\title{
Self-Replenishing Vascularized Fouling-Release Surfaces
}

\section{Citation}

Howell, Caitlin, Thy L. Vu, Jennifer J. Lin, Stefan Kolle, Nidhi Juthani, Emily Watson, James C. Weaver, Jack Alvarenga, and Joanna Aizenberg. 2014. "Self-Replenishing Vascularized Fouling-Release Surfaces." ACS Applied Materials \& Interfaces 6 (15) (August 13): 13299-13307. doi:10.1021/am503150y.

\section{Published Version}

doi:10.1021/am503150y

\section{Permanent link}

http://nrs.harvard.edu/urn-3:HUL.InstRepos:27738666

\section{Terms of Use}

This article was downloaded from Harvard University's DASH repository, and is made available under the terms and conditions applicable to Open Access Policy Articles, as set forth at http:// nrs.harvard.edu/urn-3:HUL.InstRepos:dash.current.terms-of-use\#OAP

\section{Share Your Story}

The Harvard community has made this article openly available.

Please share how this access benefits you. Submit a story.

\section{Accessibility}




\title{
Self-replenishing vascularized fouling-release surfaces
}

Caitlin Howell, ${ }^{a{ }^{*}}$ Thy L. Vu, ${ }^{a b}$ Jennifer J. Lin, ${ }^{a b}$ Stefan Kolle, ${ }^{a b}$ Nidhi Juthani, ${ }^{a b}$ Emily Watson, ${ }^{a b}$ James $C$. Weaver, ${ }^{a}$ Jack Alvarenga, ${ }^{a}$ and Joanna Aizenberg ${ }^{a b}{ }^{*}$

${ }^{a}$ Wyss Institute for Biologically Inspired Engineering, 60 Oxford Street, Cambridge, MA, 02138

${ }^{\mathrm{b}}$ School of Engineering and Applied Sciences, Harvard University, 9 Oxford Street, Cambridge, MA, 02138

*Corresponding authors: chowell@ seas.harvard.edu, jaiz@ seas.harvard.edu

\begin{abstract}
Inspired by the long-term effectiveness of living antifouling materials, we have developed a method for the self-replenishment of synthetic biofouling-release surfaces. These surfaces are created by either molding or directly embedding 3D vascular systems into polydimethylsiloxane (PDMS) and filling them with a silicone oil to generate a non-toxic oil-infused material. When replenished with silicone oil from an outside source, these materials are capable of selflubrication and continuous renewal of the interfacial fouling-release layer. Under accelerated lubricant loss conditions, fully-infused vascularized samples retained significantly more lubricant than equivalent non-vascularized controls. Tests of lubricant-infused PDMS in static cultures of the infectious bacteria Staphylococcus aureus and Escherichia coli as well as the green microalgae Botryococcus braunii, Chlamydomonas reinhardtii, Dunaliella salina, and Nannochloropsis oculata showed a significant reduction in biofilm adhesion compared to PDMS and glass controls containing no lubricant. Further experiments on vascularized versus nonvascularized samples that had been subjected to accelerated lubricant evaporation conditions for up to $48 \mathrm{~h}$ showed significantly less biofilm adherence on the vascularized surfaces. These results demonstrate the ability of an embedded lubricant-filled vascular network to improve the longevity of fouling-release surfaces.
\end{abstract}

\section{KEYWORDS}

Fouling-release surfaces; anti-fouling; self-replenishing; PDMS; bacteria; algae 


\section{INTRODUCTION}

Biofouling is a dynamic, complex process. In nature, fouling organisms have developed extremely effective strategies to attach themselves to a wide variety of surfaces under diverse and frequently changing environmental conditions. For human beings, such effectiveness and adaptability of fouling organisms has proven to have significant negative economic and environmental consequences across a wide variety of areas. Biofilm formation on medical devices is responsible for half of all healthcare-associated infections, ${ }^{1}$ costing U.S. hospitals between $\$ 28$ and $\$ 45$ billion annually ${ }^{2}$ and unquantifiable loss in quality of life for many patients. In ships, biofouling can result in power penalties of anywhere between $10 \%$ and $86 \%{ }^{3}$ and has been estimated to cost between \$180-260 million per year for the U.S. Navy fleet alone. ${ }^{4}$ Biofouling also impacts food packaging and storage, industrial-scale growth and release of algae for biofuels and other products, and water purification systems, to name just a few. ${ }^{5-8}$

Many strategies have been developed to reduce the detrimental impacts of biofouling. Current approaches generally rely on either chemical modifications, structural modifications, or some combination of the two. Chemical approaches include hydrophilic coatings such as polyethylene glycol (PEG) ${ }^{9,10}$ that prevent nonspecific adhesion of bacteria and proteins, as well as substrates that incorporate or release materials toxic to organisms, such as quaternary ammonium salts and silver nanoparticles in medical environments, ${ }^{11}$ or tributyltin (TBT) and tributyltin oxide (TBTO) in marine settings. ${ }^{12}$ Physical approaches typically use micro- or nanoscale surfaces to reduce organism contact area and adhesion strength. ${ }^{13-16}$ One such method, slippery liquid-infused porous surfaces (SLIPS), makes use of a surface-immobilized omniphobic lubricant layer to prevent the adherence of fouling organisms as well as other materials. ${ }^{17-21}$ Yet while many of these treatments show promise on short timescales, nearly all will begin to fail over extended periods. Chemical coatings are subject to degradation, depletion, or desorption, ${ }^{22}$ and physical modifications can be structurally damaged or suffer liquid infiltration. Furthermore, in many cases fouling organisms themselves adapt by generating a conditioning layer of proteins or polysaccharides to mask and condition the underlying surface. ${ }^{22,23}$

Fouling-release coatings are an alternative approach that has the potential to be used over longer periods of time. Currently used in marine environments as non-toxic alternatives to biocidal paints, ${ }^{12,24,25}$ these coatings are based on the concept that physical removal of fouling agents appears to be easiest from materials with low elastic moduli and surface free energies between 20 and $30 \mathrm{~mJ} / \mathrm{m}^{2},{ }^{24,26}$ such as silicones. Fouling organisms such as barnacles, algae spores, and bacteria can therefore be removed from fouling-release surfaces with the application of relatively low hydrodynamic forces. ${ }^{27}$ It has also been observed that the addition of some oils to silicone polymer coatings prior to curing can further enhance the fouling-release capabilities of these surfaces ${ }^{28}$ and decrease fouling levels even after two years in marine field tests. ${ }^{29}$ Yet even these materials will eventually become fouled. 
In nature, antifouling strategies adopted by various plants and animals not only successfully prevent the attachment and proliferation of fouling organisms, but they continue to do so over weeks, months, and even years, stopping only when the organism is dead. Adaptation such as complex nanoscale surface topographies on the skin of sharks and pilot whales, coatings of the fish scales with the layer of mucus, or the production of natural biocidal chemicals on the fronds of macroalgal species, e.g. Delisea pulchra, allow these organisms to remain clean over extended periods of time. ${ }^{30}$ Yet although much effort has been put into duplicating these effects by mimicking surface structure or chemical composition of these natural systems, no synthetic antifouling material has proven to be as effective as shark and fish skin or algae fronds when it comes to long-term functionality. ${ }^{30}$ The reason for this is at least partly due to the fact that surfaces on living animals and plants are dynamic, just as the organisms that try to foul them. Living surfaces can be constantly sloughed off and replenished to remove or discourage biofoulers, as opposed to static surfaces which can almost always be overcome given enough time. Some previous work has been done to try to mimic dynamic living surfaces by developing skin-like coatings which dissolve in marine environments to release fouling agents. ${ }^{31}$ However, many technical challenges related to the durability and scale-up of these types of materials remain.

Here, we describe the creation and initial testing of fouling-release surfaces made selfreplenishing through the incorporation of a vascular system as an internal reservoir. We start from a common silicone-based fouling-release material (polydimethylsiloxane [PDMS]), and coat it with silicone oil to improve its release properties. Finally, we fill the underlying vascular network with more silicone oil lubricant, which can diffuse through the solid PDMS to continually replenish the interfacial lubricating layer. We describe two methods for fabricating these vascular networks and demonstrate capacity of the system for lubricant retention and replenishment in accelerated tests. We further show that lubricant-infused silicone polymers show excellent fouling-release properties against bacterial monocultures and algae mixed cultures, and that these properties can be retained over time through the incorporation of lubricant-containing vascular networks. This work represents the next step toward the development of true bio-inspired "living" non-toxic antifouling coatings.

\section{EXPERIMENTAL SECTION}

Fabrication of PDMS Vascular Systems (Figure 1). Sylgard® 184 silicone elastomer (Dow Corning Corporation, Midland, MI) was used to fabricate all samples used in the experiments described. The base to curing agent was combined in a 10:1 ratio and mixed in a Thinky planetary centrifugal mixer (Thinky Corporation, Tokyo, Japan) at $2000 \mathrm{rpm}$ for $1 \mathrm{~min}$, then again at $2200 \mathrm{rpm}$ for $1 \mathrm{~min}$.

Encased Vasculature. Vascularized masters were fabricated by 3D printing using a Stratasys Connex500 3D printer (Stratasys Ltd., Eden Prairie, MN) with VeroBlack polyjet resin. 2D images of leaf vascular systems were employed as the original input files. Prior to molding, the masters were kept at $100^{\circ} \mathrm{C}$ for $24 \mathrm{~h}$. Uncured PDMS was then poured onto the $3 \mathrm{D}$ printed 
master, degassed under vacuum, and allowed to cure at $70^{\circ} \mathrm{C}$ for at least $2 \mathrm{~h}$. Once curing was complete, the mold was carefully separated from the master. Finally, the vasculature was encased by chemically bonding a second sheet of PDMS over the mold by applying oxygen plasma (30 s) followed by heat treatment $\left(2 \mathrm{~h}\right.$ at $70^{\circ} \mathrm{C}$ ) to both surfaces (Figure $1 \mathrm{~B}(1)$ ).

Embedded Vasculature. Channels were embedded into PDMS according to procedures developed by Lewis et al. ${ }^{32}$ Briefly, a $20 \%$ (w/v) solution of Pluronic F127 (Sigma-Aldrich, St. Louis, MO) dissolved in water was heated until it became a gel (normally around $70^{\circ} \mathrm{C}$ ) and manually embedded into uncured, degassed PDMS in desired channel patterns. The PDMS was then allowed to cure completely at $70^{\circ} \mathrm{C}$. Finally, the PDMS samples were cooled to $4{ }^{\circ} \mathrm{C}$ to cause the Pluronic F127 solution within the channels to become liquid. The liquid was then evacuated using an in-house vacuum system, leaving empty, embedded channels (Figure 1B(2)).

Lubricant Infusion. The channels in the vascularized PDMS were filled with Momentive Element 14* 10A silicone oil (Momentive, Albany, NY). These samples, as well as solid PDMS for controls and initial biological tests, were completely immersed in a silicone oil bath for 2-20 days (depending on initial thickness) until the mass of the samples no longer increased and full infusion of the lubricant into the PDMS molecular structure had occurred.

Lubricant Replenishment Tests. Accelerated testing of lubricant replenishment in cylindrical vascularized samples compared to non-vascularized controls was performed by placing fully-infused samples in an oven at $70^{\circ} \mathrm{C}$. Cylindrical embedded vascularized samples and equivalent controls were used due to their smaller size and, therefore, faster rate of infusion. In the vascularized samples, the free silicone oil took up approximately $17 \%$ of the entire sample volume. Samples were gently wiped prior to the experiment to remove any excess lubricant from the surface. The masses of the samples were then taken daily using a Mettler Toledo Xs205 DualRange analytical balance to measure lubricant loss via evaporation. Before weights were taken, the channels of the vascularized samples were refilled using a small-gauge syringe inserted through the encasing PDMS into the channel in one corner of the sample. The air was allowed to escape via a second small puncture in the opposite corner. This was done in order to mimic a system with a continuous lubricant supply.

Bacterial Biofouling Tests. Liquid stock solutions of Staphylococcus aureus strain SC01 in tryptic soy broth (TSB) medium and Escherichia coli strain J96 in M63 medium with densities of approximately $10^{8}$ cells/mL were prepared. S. aureus and E. coli were chosen due to their role as an infection-causing agents in many healthcare settings. ${ }^{1}$ The stock solutions were diluted 1:100 (v/v) in TSB biofilm medium (TSB with 1.5\% (w/v) NaCl) in petri dishes containing samples of glass, non-infused PDMS, and lubricant-infused PDMS (nonvascularized). Cultures were then incubated at $37^{\circ} \mathrm{C}$ for $48 \mathrm{~h}$.

Following incubation, the substrates were gently removed from the biofilm medium. Samples for biofilm coverage analysis were immediately stained by incubating in a $0.1 \%(\mathrm{w} / \mathrm{v}$ ) solution of crystal violet in water for $10 \mathrm{~min}$, gently rinsed in deionized, distilled water to remove excess stain, then dried and photographed. Samples for colony-forming unit (CFU) counts were sonicated for $5 \mathrm{~min}$ in 1x phosphate buffered saline (PBS) to remove the bacteria 
from the surfaces, then diluted and plated out on TSB agar. For both biofilm coverage and CFU counts at least five replicates per treatment were prepared.

For bacterial biofouling tests on vascularized PDMS versus controls, samples were kept under static conditions at $70^{\circ} \mathrm{C}$ for $1,2,4,8,24$, and $48 \mathrm{~h}$ to cause lubricant loss through evaporation. Both unlubricated samples and fully lubricated samples that had not been exposed to evaporation were included as controls. The channels of the vascularized samples were kept continuously filled in the same manner used for the lubricant replenishment tests. Samples were then immersed in bacterial medium for $48 \mathrm{~h}$, removed, and stained with crystal violet as described above.

Algae Biofouling Tests. Stock solutions of non-axenic Botryococcus braunii (UTEX number 2441) 3 $^{33}$ and Chlamydomonas reinhardtii (UTEX number 89 ) from the University of Texas Culture Collection were grown in Basal Medium and Bristol Medium, respectively. Dunaliella salina cultures were provided by the Hastings Lab at Harvard University and maintained in Guillard f/2 marine water enrichment solution (50x) (G0154, Sigma-Aldrich, St. Louis, MO). Nannochloropsis oculata cultures were provided by Solix Biosystems and grown in a modified $\mathrm{f} / 2$ medium with vitamins omitted.

Once a density of approximately $10^{7}$ algal cells per $\mathrm{mL}$ was reached, these cultures were diluted 1:5 in petri dishes containing fresh medium. These dishes contained non-infused and lubricant-infused PDMS (non-vascularized) test surfaces created by spin-coating PDMS onto flat glass substrates, as well as glass controls. Cultures were incubated under a set of Sun Blaze T5HO fluorescent light fixtures (Sunlight Supply, Inc., Vancouver, WA). The lamps were on timers to provide $16 \mathrm{~h}$ of light followed by $8 \mathrm{~h}$ of darkness. Surface temperatures were monitored throughout the experiment and did not exceed $25^{\circ} \mathrm{C}$. There were at least five replicates per treatment.

After 12 days, the substrates were gently lifted at one end to attach a clip connected to a dip-coater via a nylon cord. The substrates were then lifted out of the culture medium at a controlled rate of $0.5 \mathrm{~mm} / \mathrm{s}$. Once the substrate was completely clear of the medium it was photographed for image analysis. Immediately after photographing, the samples were processed for chlorophyll $a$ as a proxy for estimated biofilm mass. Each sample was placed into a $600 \mathrm{~mL}$ beaker filled with $300 \mathrm{~mL}$ DIW and sonicated for $2 \mathrm{~min}$. The resulting solution biomass was then vacuum-filtered using a ceramic funnel with Whatman 1 filter papers. Each filter paper was immersed in $5 \mathrm{~mL}$ DMSO and was heated overnight at $70^{\circ} \mathrm{C}$. A $200 \mu \mathrm{L}$ volume of this solution was then measured for absorbance at 665 and $750 \mathrm{~nm}$. The resulting chlorophyll $a$ values were then determined using the following equation for each sample: ${ }^{34}$

$$
C_{\mathrm{a}}=\left(D_{\mathrm{b}}-D_{\mathrm{a}}\right) \cdot[R /(R-1)] \cdot(v / l) \cdot\left(10^{3} / \alpha_{\mathrm{c}}\right),
$$

where $C_{\mathrm{a}}$ is the concentration of chlorophyll $a$ in $\mathrm{mg} / \mathrm{mL}$ solvent, $D_{\mathrm{b}}$ and $D_{\mathrm{a}}$ are the optical densities before and after acid addition, respectively, $\alpha_{c}$ is the specific absorption coefficient for chlorophyll $a, v$ the volume of solvent used to extract the sample, and $l$ the path length of the 
spectrophotometer in $\mathrm{cm} . R$ is defined as $D_{\mathrm{b}} / D_{\mathrm{a}}$ for pure chlorophyll $a$. The values obtained at $750 \mathrm{~nm}$ were subtracted from those at $665 \mathrm{~nm}$ to account for sample turbidity. ${ }^{34}$

Toxicity tests. Experiments to determine toxicity were conducted on B. braunii cultures as these were considered likely to be more sensitive to adverse effects from silicone oil (suffocation, light interference) due to their need to photosynthesize. ${ }^{27}$ A $500 \mathrm{~mL}$ volume of Basal Medium containing a 1:5 ratio of B. braunii stock was placed into 1L Erlenmeyer flasks either with or without $10 \%$ (v/v) of the additional silicone oil. The cultures were then bubbled with air to ensure continuous mixing. There were five replicates per treatment.

Every three days, approximately $1 \mathrm{~mL}$ of the culture medium was removed for analysis. Of this, a $500 \mu \mathrm{L}$ aliquot was incubated with $10 \mu \mathrm{M}$ Sytox Green Nucleic Acid Stain (Life Technologies, Carlsbad, CA) for $10 \mathrm{~min}$, then visualized using a Zeiss microscope with an excitation wavelength at $470 \mathrm{~nm} .{ }^{35}$ Using this procedure, all dead cells were stained green. Live algae produced red autofluorescence due to their chlorophyll $a$ content. Five images were taken per sample. Cells were counted using MatLab (Mathworks, MA, USA) prior to statistical analysis. A $200 \mu \mathrm{L}$ aliquot of the remaining material was used to measure optical density at 683 nm using a SpectraMax 384 plate reader (Molecular Devices, LLC, Sunnyvale, CA).

Biofilm coverage analysis. After removal from the liquid medium (and crystal violet staining in the case of the bacterial fouling tests), all test surfaces were photographed with a Canon EOS Rebel T4i camera (Melville, NY). Images were analyzed by thresholding such that biofilm-covered areas became white and the clear areas became black. This allowed for the calculation of the area that was thresholded and hence the area of the biofilm, which was divided by the area of the sample to determine the percent coverage. Image analysis was performed either using ImageJ or MatLab (Mathworks, MA, USA).

Statistics. For image analysis, the percent area values between 0 and $100 \%$ were first arcsine transformed to change the distribution from binomial to nearly normal. ${ }^{36}$ Two-tailed $\mathrm{t}$ tests or univariate ANOVAs with Tukey post-hoc test were then performed to determine statistical significance between the control and lubricant-infused treatments. Live cell counts in the toxicity tests were analyzed using a repeated-measures two-way analysis of variance. All statistical analyses were performed using IBM SPSS Statistics version 20 (IBM, Armonk, NY). A value of $\mathrm{P}<0.05$ was considered significant.

\section{RESULTS AND DISCUSSION}

Fabrication of PDMS Vascular Systems. Samples of either vascularized or nonvascularized lubricant-infused PDMS were prepared as schematically depicted in Figure 1A. Vascular systems were fabricated either as encased channels or embedded channels as described in the materials and methods section. Figure $1 \mathrm{~B}(1)$ shows the process of fabricating an encased vasculature system based on the vein structure of a leaf. The desired 2D pattern was converted into a black and white vector drawing, imported into SolidWorks (Dassault Systems, Waltham, MA), then 3D printed to make a master. The master was used to mold the vascular pattern into PDMS. The final mold was then covered in a second sheet of PDMS to enclose the vascular 
channels. With this fabrication method it was possible to create complex channel systems over a potentially wide area (depending on the size of the 3D printed master). However, it was noted that great care needed to be taken when attaching the covering PDMS sheet to the final mold. Any incomplete chemical bonding between the two PDMS pieces, due to such factors as overexposure to plasma or presence of surface contamination, was found to result in delamination upon lubricant infusion.

Embedding the vascular system directly into uncured PDMS was explored as a way to avoid delamination, as well as a way to make multiple smaller samples in parallel for further characterization. Lewis et $a l^{32}$ developed the method of using Pluronic F127 for embedding 3Dprinted channel systems into polymers, such as PDMS, for culturing cells. This compound, when mixed with water at a $20 \% \mathrm{w} / \mathrm{v}$ ratio, produces a solution which is a gel above $25^{\circ} \mathrm{C}$, but a liquid at around $4^{\circ} \mathrm{C}$. By exploiting this property it was possible to manually draw channels into PDMS using the gel-phase Pluronic at $25^{\circ} \mathrm{C}$, cool it to $4^{\circ} \mathrm{C}$ and remove the then liquid-phase Pluronic using vacuum, followed by re-filling the channels with silicone oil lubricant. Using this procedure, a variety of simple vascular systems could be created (Figure 1B(2)).

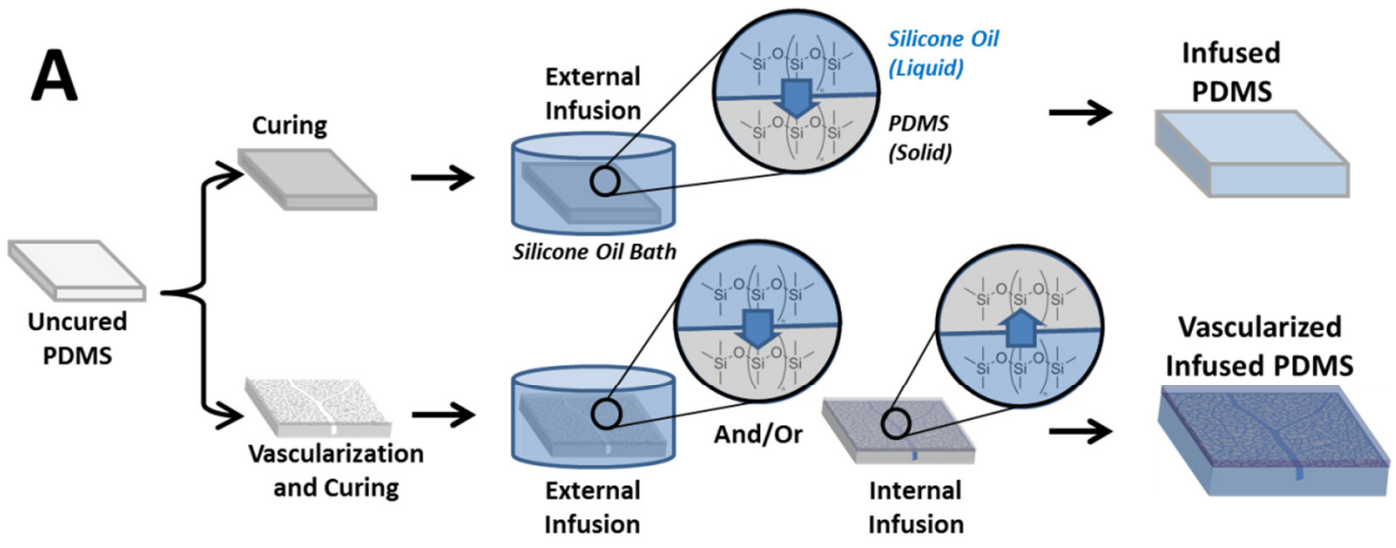

\section{B Vascularization Methods:}
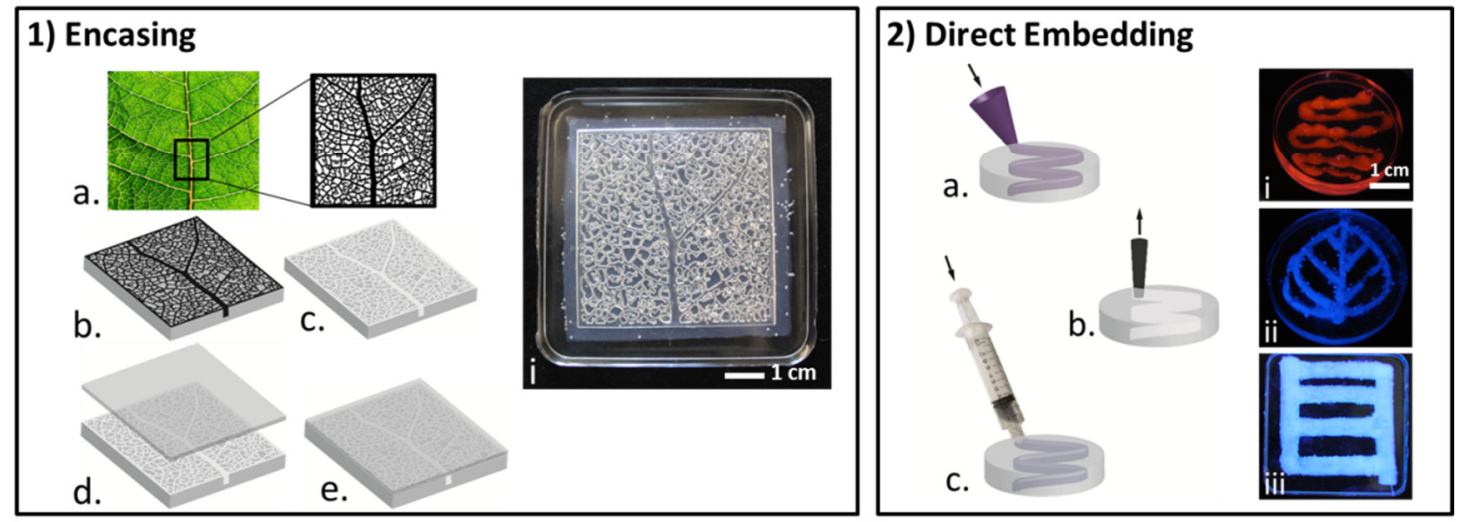

Figure 1. (A) Schematic of the process to make either vascularized or non-vascularized silicone-oilinfused PDMS. For simple infused PDMS (upper row), cured PDMS is placed in a bath of silicone oil, which diffuses into the PDMS solid. For vascularized PDMS, the vascular pattern is created before curing. The sample is then infused externally with silicone oil in the same manner as the non- 
vascularized PDMS, or internally through filling the vascular network, or both. (B) Methods of creating vascular networks within PDMS: 1) An encased network is created using a 3D mold (a) to create the pattern in PDMS (b). The mold is removed from the cured PDMS (c) and the pattern is covered with a second sheet of PDMS ( $\mathrm{d}$ and e), which is chemically bonded to the pattern using $\mathrm{O}_{2}$ plasma. (i) An image of a 3D leaf vasculature network after encasing. 2) An embedded network is created following the procedures developed by Lewis et al. ${ }^{32}$. (a) A pattern of $20 \%$ w/w Pluronic F127 gel at $25^{\circ} \mathrm{C}$ is embedded in uncured PDMS. (b) The PDMS is allowed to cure, cooled to $4{ }^{\circ} \mathrm{C}$, and the liquid Pluronic is evacuated. (c) The channel is refilled with silicone oil. An image of fluorescently dyed silicone oil in (i) a sinusoidal channel, (ii) a leaf-shape network, and (iii) a linear network.

Lubricant Replenishment Tests. Experiments were conducted on embedded vascular systems and non-vascularized controls to compare their ability to secrete the lubricant from the channels to the surface upon interfacial lubricant loss. Evaporation at $70^{\circ} \mathrm{C}$ was chosen as the test method of lubricant removal from the surfaces as opposed to wiping or depletion via water flow for two reasons: first, it provided a more even removal than physical wiping without damaging the surfaces; and second, it removed lubricant from the surfaces more quickly than the low-flow conditions these materials would be exposed to in medical devices or stationary marine applications thus offering a more suitable accelerated test. Evaporation at elevated temperatures would also be expected to be the primary route of lubricant loss if these materials were exposed to alternating marine/open air conditions such as coatings for dock pillars exposed to tides, for example, as previous experiments on radiolabeled oil loss from PDMS samples showed less than $1 \%$ loss over 1 year under simulated marine conditions when not exposed to evaporation. ${ }^{29}$

The channels containing free oil took up approximately $17 \%$ of the total volume of the vascularized samples, and samples saturated with the lubricant oil had masses approximately 1.3 greater than unsaturated samples. The results of accelerated evaporation tests on these initially saturated samples gathered over 11 days are shown in Figure 2. The vascularized PDMS samples lost $4.6 \%( \pm 2.5 \%)$ of their lubricant volume (with continual lubricant replenishment), while the non-vascularized control lost $13.5 \%( \pm 1.2 \%)$. There was a clear significant difference in the amount of lubricant lost between the two $(\mathrm{P}<0.01)$. After 24 days, this difference had widened to $6.5 \%( \pm 3.7 \%)$ lubricant loss in the vascularized samples compared to $20.8 \%( \pm 1.2 \%)$ loss in the controls. 


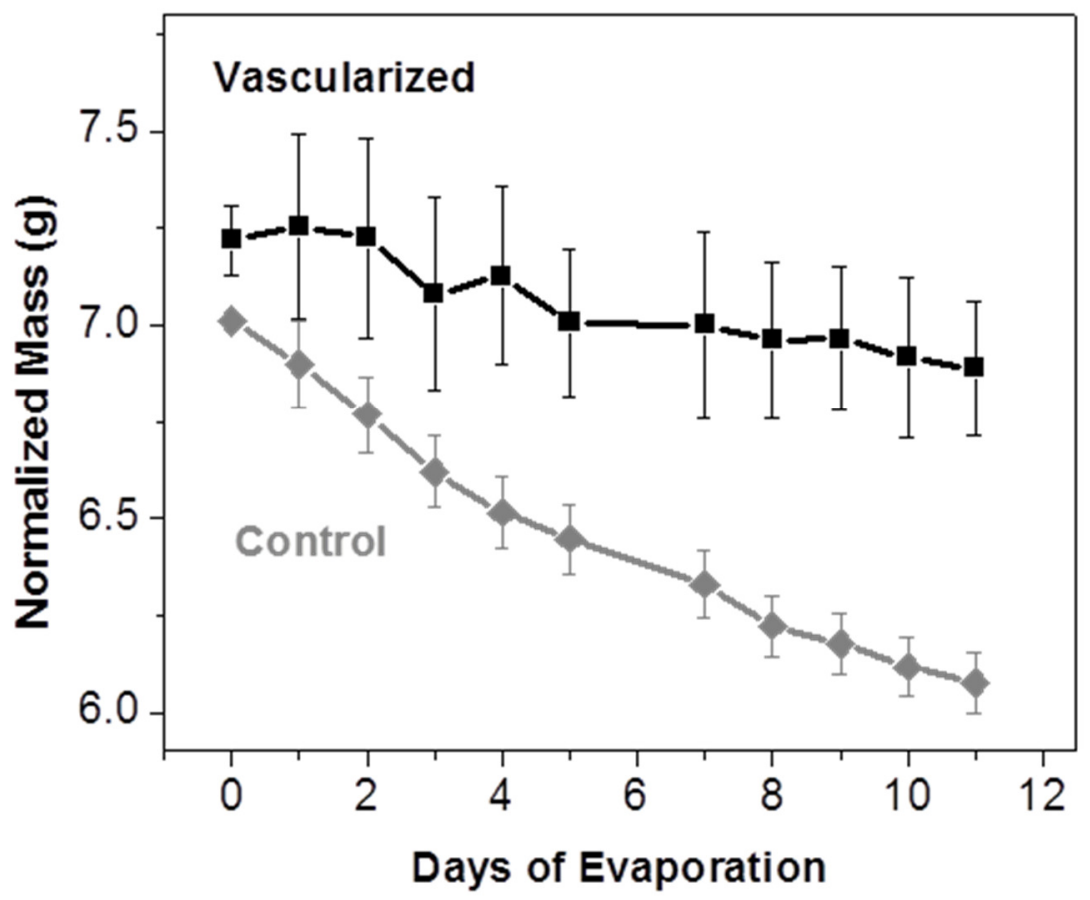

Figure 2. Mass of saturated lubricant-infused PDMS samples containing an embedded vascular network (squares) versus a sample with no vascular network (diamonds) kept at $70^{\circ} \mathrm{C}$ for 11 days. The data were normalized to the dry mass of the samples and error bars represent standard deviation. The control lost mass due to lubricant evaporation more rapidly than the channel system in this accelerated test.

These results show that the presence of a vascular system slows lubricant loss in PDMS samples via evaporation, providing on-demand replenishment of the lubricant in the system that can counteract lubricant losses from the surface.

Biofouling Tests. Previous studies have shown that the incorporation of free silicone oil into silicone polymer coatings can help increase their fouling resistance in marine settings. ${ }^{28,29}$ However, these samples were generally created as one-pot coatings already containing free oil rather than as oil added to the polymer after curing by post-fabrication swelling or as oil diffusing to the interface from the vascularized network. To ensure that the samples saturated with oil post-fabrication would also function efficiently as fouling-release surfaces against model organisms, we tested fully-infused PDMS substrates against static cultures of the infectious bacterium $S$. aureus $^{1}$ as well as against the model microalga B. braunii. ${ }^{33,37}$ These test were performed first on non-vascularized samples as lack of evaporation or other lubricant-removing forces made the inclusion of vasculature unnecessary.

The results of the static tests against $S$. aureus are shown in Figure 3. Both glass and non-infused PDMS were used as positive controls to demonstrate the effects of biofilm formation on hydrophilic and hydrophobic surfaces, respectively. Figure 3A and B shows that both of these surface types fail to resist biofilm attachment, resulting in biofilm coverages between $73.9 \%$ and $64.8 \%\left( \pm 7.5 \%\right.$ ) (Figure $3 \mathrm{C}$ ), and CFU counts of $6.5 \times 10^{8}$ and $10.7 \times 10^{8}$ 
cells $/ \mathrm{cm}^{2}$ (Figure 3D) for glass and untreated PDMS, respectively. The lubricant-infused PDMS samples, in contrast, showed no visible adherent biofilms upon being removed from the culture medium, resulting in surface coverage values of $0.1 \%( \pm 0.1 \%)$ after crystal violet staining. $\mathrm{CFU}$ counts for these samples yielded values of $4.8 \times 10^{5}$ cells $/ \mathrm{cm}^{2}$, three orders of magnitude lower than the control samples. These results demonstrated that lubricant-infused PDMS can be significantly more effective as fouling-release surfaces against biofilms of $S$. aureus compared to even low-adhesion surfaces of PDMS or glass.

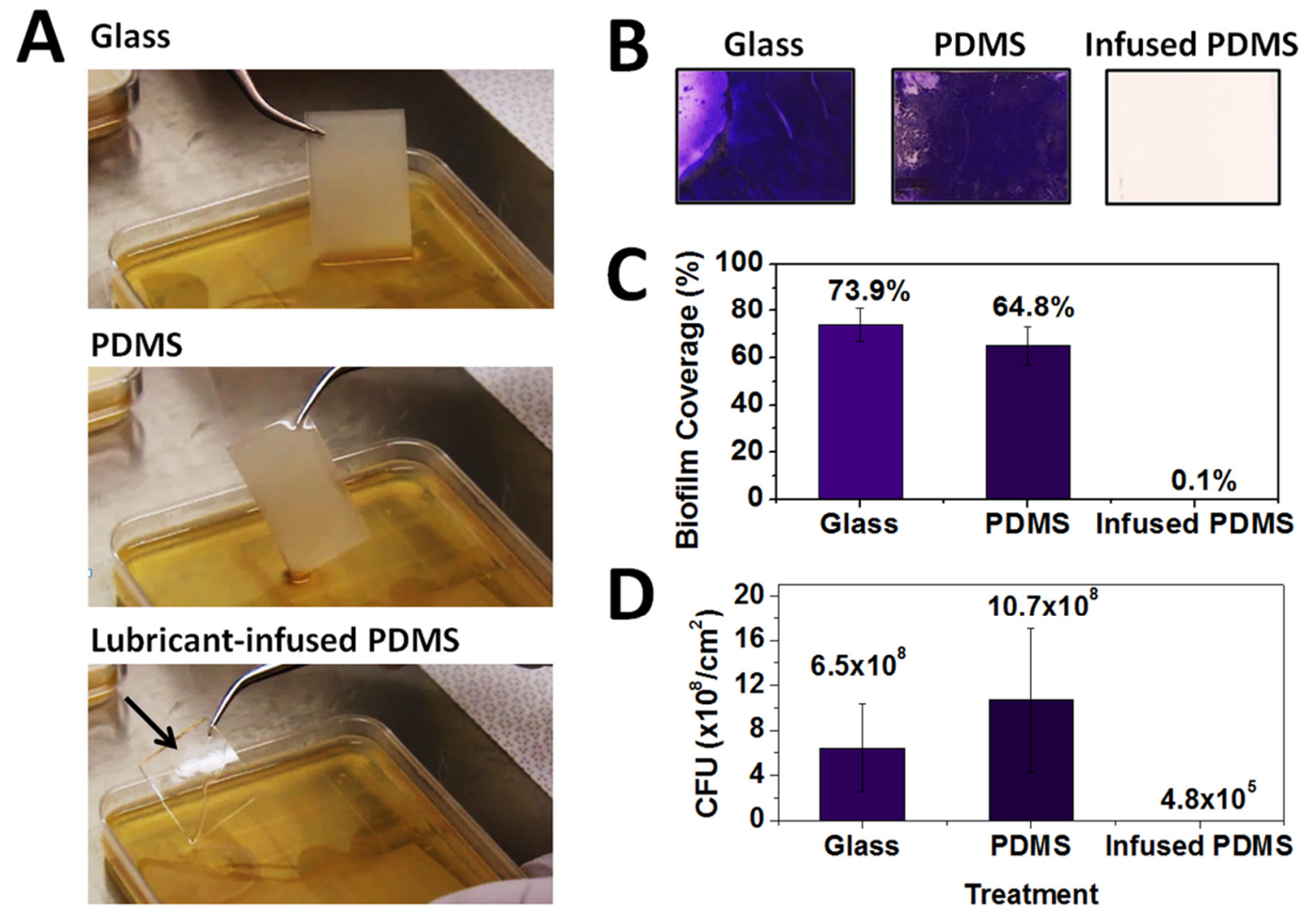

Figure 3. (A) Removal of glass, PDMS, and lubricant-infused PDMS from a solution of S. aureus after $48 \mathrm{~h}$ of growth. The black arrow indicates the location of the infused PDMS sample. Note that the controls become cloudy due to the biofilm overgrowth, while the sample that was infused with the oil post fabrication is completely clear and transparent. (B) Representative samples after staining for adherent biofilm with crystal violet. A black line is added to outline the infused PDMS sample due to its nearcomplete transparency. (C) Image analysis results (\% biofilm coverage) of the surfaces. (D) CFU counts showed a decrease of at least three orders of magnitude in the lubricant-infused PDMS samples compared to the control PDMS and glass samples.

Biofouling tests on substrates in static cultures of the microalgae B. braunii showed similar results. Upon removal of the substrates from the culture medium, the biofilm peeled off nearly completely of the lubricant-infused samples, while it remained firmly attached on the control samples. This effect was most striking on glass substrates that had been half-coated in lubricant-infused PDMS, as shown in Figure 4A. As these samples were removed from the culture solution, the biofilms began to peel away from the surface as soon as the lubricant- 
infused coating was reached. In fact, it is interesting to note that the biofilm was so poorly adhered to the lubricant-infused surface compared to the glass surface that the film was almost perfectly ripped in half at the glass/lubricant-infused PDMS boundary. This effect is most difficult to obtain in the direction of removal shown in Figure 4A (first glass, then lubricantinfused PDMS), as the biofilm initially covered the entire surface and would have therefore offered some stability across the boundary. After removal of the surfaces, the intact algae biofilm could be observed floating on the surface of the culture medium (as indicated by a white arrow).
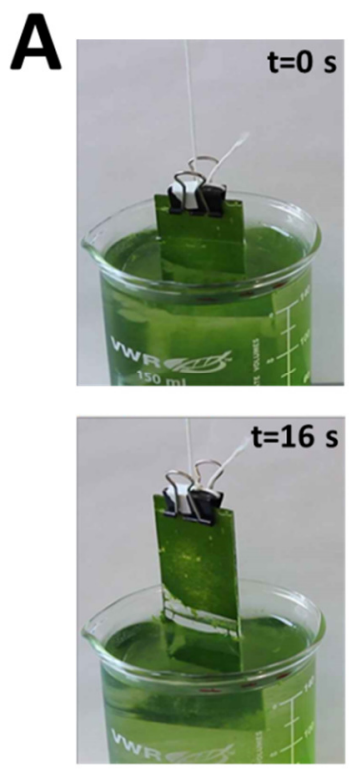
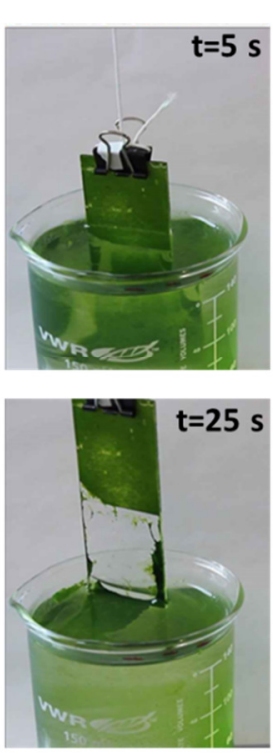
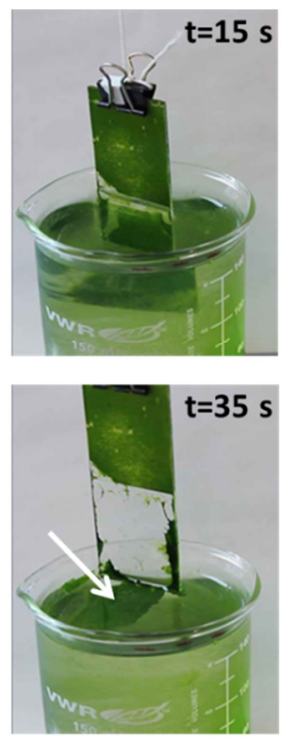
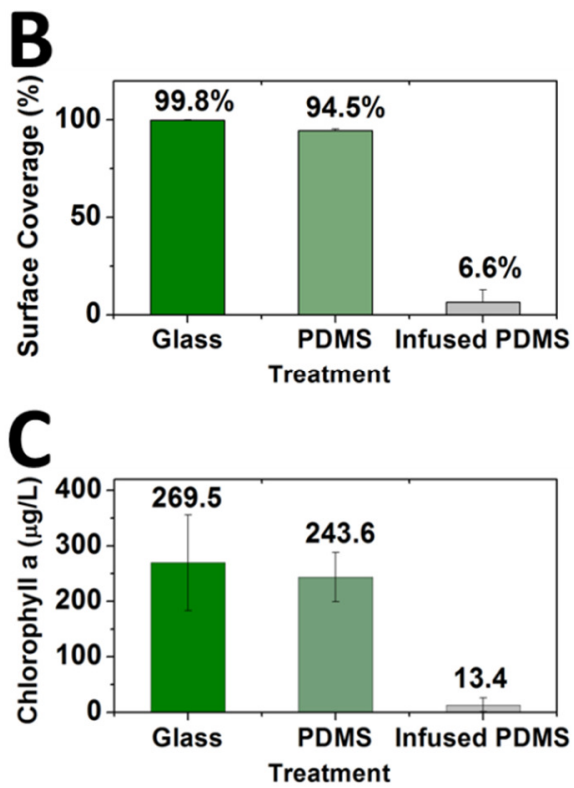

Figure 4. (A) Glass slides half-coated with lubricant-infused PDMS were incubated in culture vessels containing $B$. braunii. After 12 days, the slides were slowly removed from the vessel using a dip coater attached to the slide. At $\mathrm{t}=0 \mathrm{~s}$ and $\mathrm{t}=5 \mathrm{~s}$, the clear attachment of the algae biofilm to the untreated glass can be seen. However, at $\mathrm{t}=15 \mathrm{~s}$ the boundary between the treated and untreated class is crossed, and at $\mathrm{t}=16 \mathrm{~s}$ the biofilm begins to peel away from the surface. The arrow at $\mathrm{t}=35 \mathrm{~s}$ indicates the intact biofilm floating on the surface of the culture medium. (B) Percent biofilm surface coverage of the glass substrates, untreated PDMS substrates, and lubricant-infused PDMS substrates after removal. (C) Amount of chlorophyll $a$ remaining on the surfaces after removal.

Some adherent biofilm did remain on the edges of the lubricant-infused half of the sample. This was due to edge effects of the spin-coated PDMS providing areas of incomplete surface coverage. Such remaining material would be minimized in larger coated samples or materials made exclusively of lubricant-infused polymer, and thus would avoid the edge effect. Analysis of percent biofilm coverage for completely coated surfaces yielded values of $99.8 \%$ and $94.5 \%( \pm 0.7 \%)$ for the glass and PDMS substrates, respectively, while for the lubricant-infused PDMS surfaces this value was as low as $6.6 \%( \pm 6.4 \%)$ even with the contribution from the edge effect (Figure 4B). Quantification of the amount of chlorophyll $a$ on these surfaces gave 269.5 and $243.6 \mu \mathrm{g} / \mathrm{mL}( \pm 86.2 \mu \mathrm{g} / \mathrm{mL})$ for glass and PDMS, respectively, compared to $13.4 \mu \mathrm{g} / \mathrm{mL}$ $( \pm 12.2 \mu \mathrm{g} / \mathrm{mL}$ ) for the lubricant-infused PDMS (Figure 4C). These data confirmed that lubricant-infused PDMS was also effective at releasing mixed microalgae/bacterial biofilms. 
Furthermore, taken together with the $S$. aureus data, the results showed that for these types of lubricant-infused substrates all that was needed to remove the adherent biofilm was the small amount of force applied by passing the samples through the air/water interface.

Similar experiments were performed using the three commercially-relevant species $C$. reinhardtii, D. salina, and $N$. oculata, along with their naturally-associated bacteria, to evaluate the effectiveness of PDMS that had been infused with oil post-fabrication when exposed to different algae species. B. braunii was selected for these studies as it forms strong biofilms under static conditions and is also well known for its capacity to produce a very high lipid content within its cell walls, an important factor in the cost-effective production of biofuels, while $C$. reinhardtii, D. salina, and $N$. oculata were selected for their industrial relevance. ${ }^{38}$ Glass samples half-covered with lubricant-infused PDMS were placed in shallow petri dishes containing either static ( $C$. reinhardtii and $D$. salina) or shaking ( $N$. oculata) algae cultures. The $C$. reinhardtii and $D$. salina cultures remained green throughout the experiment, while the $N$. oculata cultures became stressed and yellowed, as was expected due to the short light path. ${ }^{39,40}$ After 12 days of growth, the samples were lifted out of the medium such that the glass control and the lubricantinfused PDMS portions of the sample were side-by-side. The same relatively clean separation of the biofilms along the glass/infused PDMS boundary observed for B. braunii (Figure 4) also occurred in these samples (Figure 5).

All three species tested showed significantly less surface coverage of biofilm on lubricant-infused surfaces compared to untreated glass control surfaces (Figure 5A). In cultures containing $C$. reinhardtii, the surface coverage was $99 \% \pm 1 \%$ for glass control surfaces compared to $16 \% \pm 21 \%$ for lubricant-infused PDMS. The high level of variation in these samples was due to the presence of edge effects (see Fig. 5B), similar to what was observed in the $B$. braunii samples (Fig. 4A). Cultures with D. salina showed biofilm surface coverage of 70 $\pm 13 \%$ for glass controls and $4 \% \pm 2 \%$ for lubricant-infused surfaces. The substrates in $N$. oculata cultures were stained with crystal violet to improve contrast upon removal. The lack of contrast initially in these biofilms was suspected to be due to the low chlorophyll $a$ and high carotenoid content, as well as the likely higher levels of bacteria that can arise in stressed algae cultures. ${ }^{39,40}$ After staining and rinsing, these surfaces showed coverages of $74 \% \pm 9 \%$ for glass controls and $2 \% \pm 2 \%$ for lubricant-infused PDMS.

Fouling Tests on Vascularized Samples. To test the ability of an incorporated vasculature to maintain these fouling-release effects in extreme lubricant-removal conditions, vascularized and non-vascularized substrates, which had been kept at $70^{\circ} \mathrm{C}$ for either $1,2,4,8$, 24 , or $48 \mathrm{~h}$ to accelerate lubricant loss via evaporation, were exposed to bacterial culture. Control samples were found to lose up to $\sim 5 \%$ of their total mass (up to $\sim 11 \%$ of infused lubricant mass) after $48 \mathrm{~h}$. The internal reservoirs of the vascularized samples were refilled at each time point using a syringe as described in the Experimental section. Following evaporation, samples were exposed to E. coli cultures for $48 \mathrm{~h}$. E. coli was chosen for these experiments instead of $S$. aureus as the former was observed to produce more strongly adherent biofilms on surfaces with minimal amounts of lubricant. This is not surprising, as previous work by Levkin 
et al. has shown that even different strains of the same bacterium can result in different biofilm attachment abilities on lubricant-infused surfaces. ${ }^{20}$
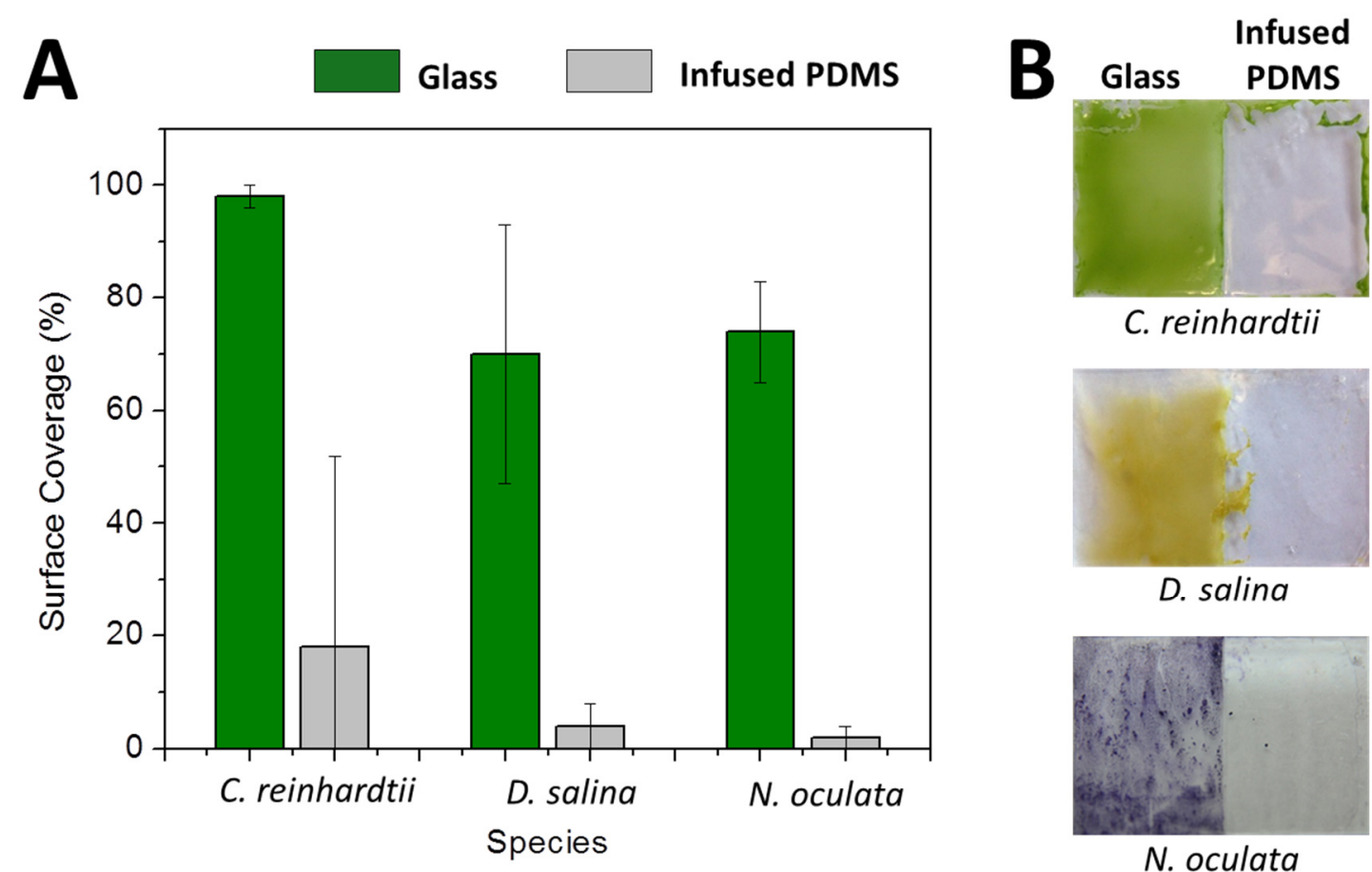

N. oculata

Figure 5. (A) Surface coverage of biofilm remaining on lubricant-infused PDMS versus glass control surfaces after incubation in and removal from the liquid medium containing C. reinhardtii, D. salina, or $N$. oculata. For all three species, there was a significant reduction in the amount of biofilm remaining on the lubricant-infused surfaces $(\mathrm{P}<0.05)$. (B) Images of the substrates used in the analysis. The biofilms of $N$. oculata and associated bacteria were dyed with crystal violet in order to improve contrast.

Figure 6 shows representative samples and their quantification for percent biofilm coverage after removal and staining with crystal violet. Unlubricated control samples (Dry) versus unevaporated samples (0h) (Figure 5A) showed results similar to what was observed for S. aureus cultures (Figure 3), e.g. a significant decrease in biofilm attachment on the lubricantinfused samples $(\mathrm{P}<0.01)$.

As the amount of time during which the samples were exposed to lubricant loss via evaporation increases, the amount of biofilm that was able to adhere to the control samples also increases, reaching a level equal to that of the unlubricated controls after $48 \mathrm{~h}$ (Figure 6A). The vascularized samples, however, show no significant increase in biofilm attachment after exposure to the same conditions due to the transport of oil from the channels to the interface. 


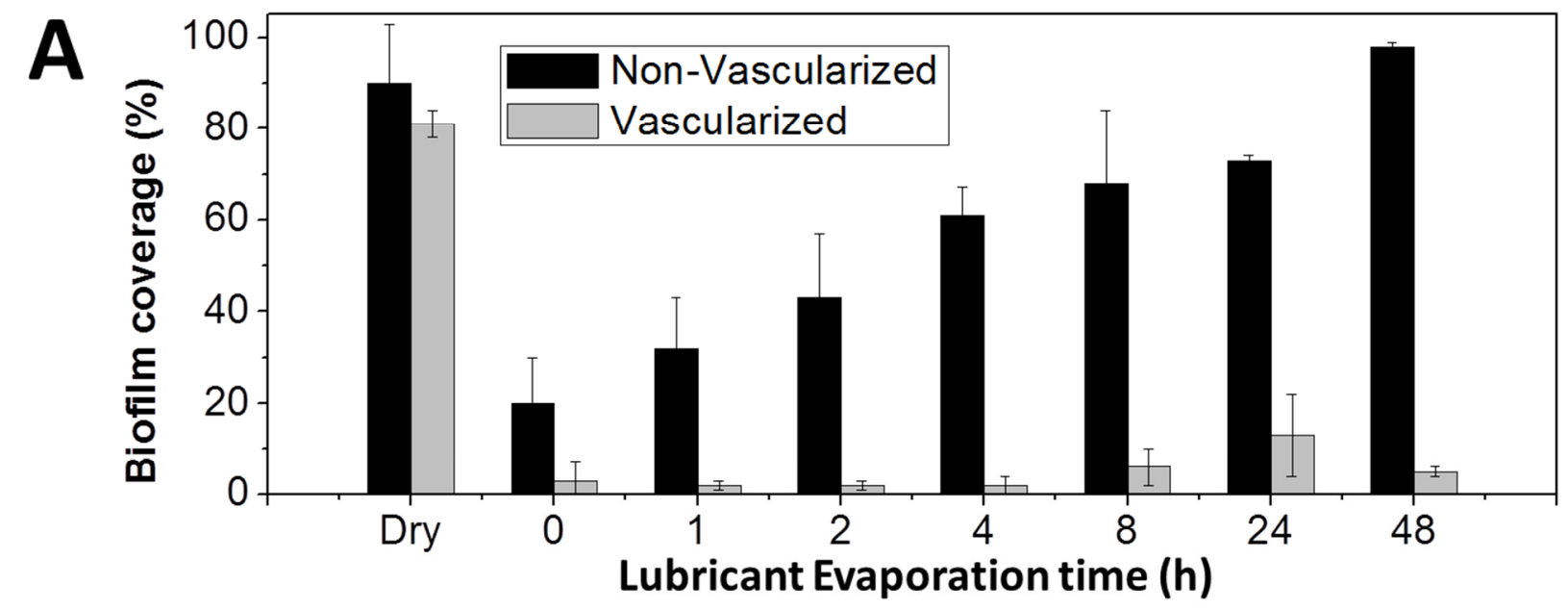

B

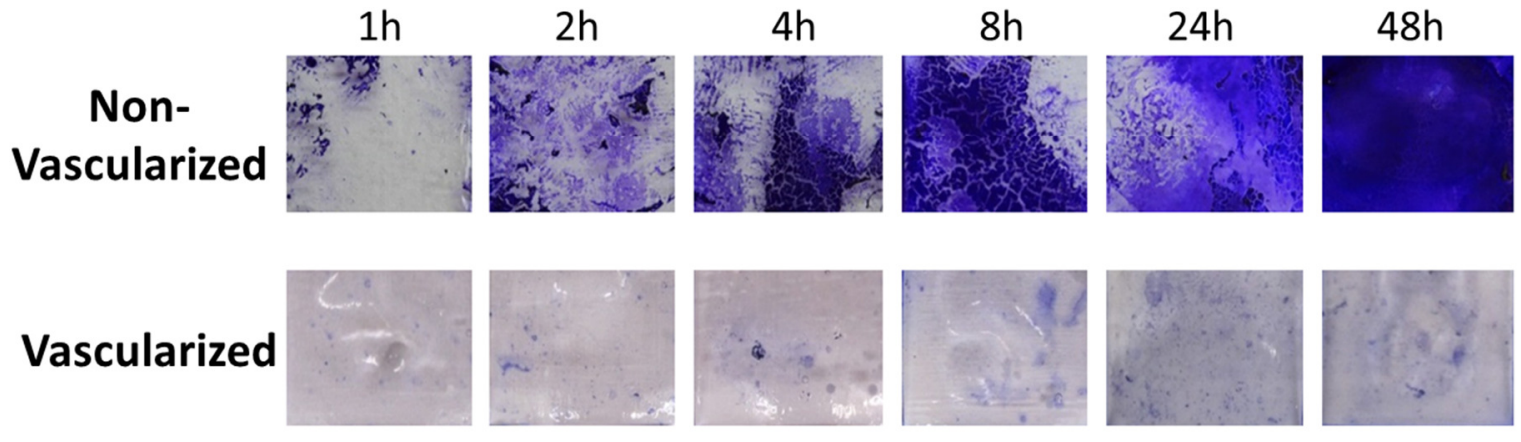

Figure 6. (A) Quantification of the amount of biofilm remaining on the surfaces compared to unlubricated controls (Dry) and samples that had undergone no lubricant evaporation (0h). The samples with embedded vasculature show significantly less adherent biofilm at all time points $(\mathrm{P}<0.05)$. (B) Representative images of control samples and samples with an embedded vascularized network after evaporation of the lubricant for 1, 2, 4, 8, 24, and $48 \mathrm{~h}$ and subsequent exposure to E. coli for $48 \mathrm{~h}$ (purple color is from crystal violet staining).

These results show that the inclusion of a vascular system can effectively prolong the fouling-release effects of lubricant-infused PDMS even at harsh conditions of accelerated lubricant removal.

Lubricant toxicity tests. Although silicone oil has been shown in multiple studies to be non-toxic to both macro- and micro-organisms in the traditional sense, ${ }^{41,42}$ there has been some concern that this low-surface-energy liquid could wrap around a single-celled organism, resulting in suffocation. ${ }^{27}$ To verify that the lubricant-infused PDMS surfaces were not showing superior biofilm release as a result of suffocation, two separate analyses were done to monitor the growth of B. braunii exposed to excess amounts of the lubricant silicone oil. B. braunii was chosen for 
these tests instead of $S$. aureus for two reasons: first, previous work has already reported similar studies on the toxicity of various other types of lubricants to bacteria ${ }^{18}$ with no effect found, and second, algae are more likely to show effects of suffocation quickly due to their need to access dissolved gases for photosynthesis. ${ }^{27}$

Figure 7 shows the growth curves of $B$. braunii in culture medium containing $10 \%$ lubricant (v/v) compared to control cultures containing no lubricant (note that $10 \mathrm{v} \%$ oil in culture represents orders of magnitude greater concentration of the lubricant than what can be released from the oil-infused polymer). Both the optical density (Figure 7A) and the total live cell count (Figure 7B) were measured; the former to reflect the changes in both the algal and naturally associated bacterial populations and the latter to show the changes in just B. braunii. Optical density measurements were taken at the experimentally determined peak absorbance of $683 \mathrm{~nm}$, in agreement with previously reported values. ${ }^{43}$ Both curves showed the expected initial lag phase followed by the exponential growth and subsequent saturation of the population. ${ }^{37}$ Logistic regressions were conducted on the optical density data to find the growth rate of the cultures. Statistical analysis showed no significant difference between the growth rate of $B$. braunii in lubricant-containing and lubricant-free control cultures across the 14-day time period. Repeated-measures two-way analysis of variance on the total live cell counts also showed no significant difference between the cell counts in lubricant-containing and control cultures over the 14-day period.

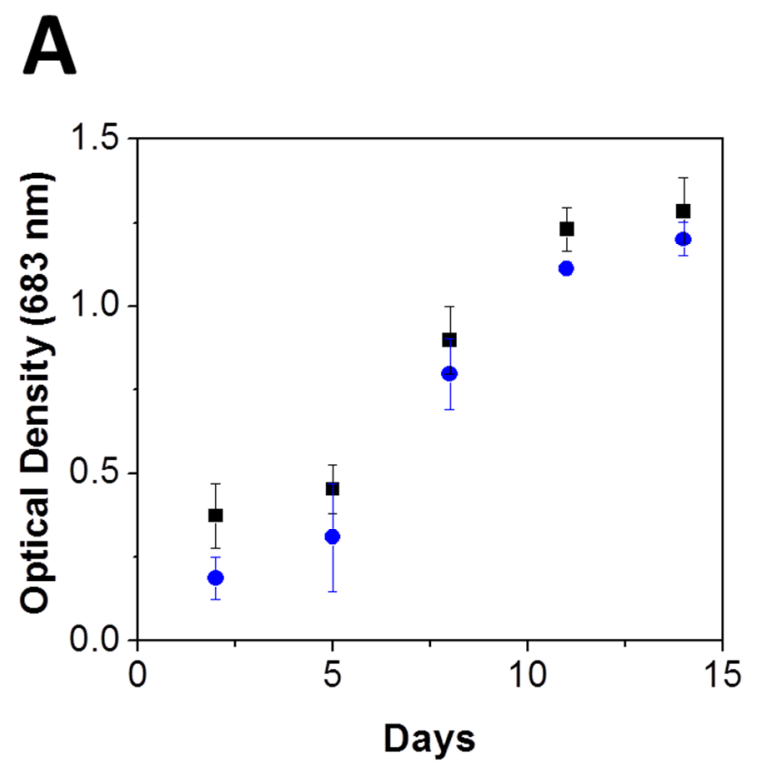

\section{B}

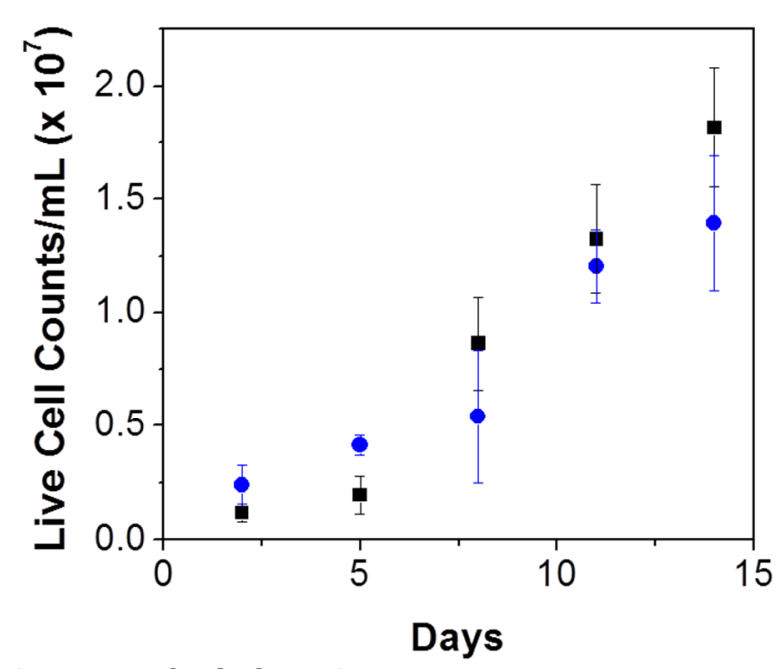

- No Lubricant - Lubricant

Figure 7. (A) Optical density measurements at $683 \mathrm{~nm}$ and (B) total live cell counts for B. braunii cultures containing $10 \%$ (v/v) lubricant (squares) and no lubricant (diamonds) over 14 days of growth. There were no significant differences in the rate of growth as indicated by these two parameters between those cultures grown with lubricant and those grown without $(\mathrm{P}<0.05)$. 
These results confirm that the apparent inability of these biofilms to adhere to lubricantinfused PDMS surfaces is not due to a lubricant toxicity or suffocation effect. Therefore, the biofilm-release process is more likely caused by the replenishment of the lubricant on the PDMS surface and its fouling-release properties, perhaps through the continual sloughing off of the surface lubricant layer.

Previous work on SLIPS exposed to cultures of Pseudomonas aeruginosa, E. coli, as well as $S$. aureus has shown that these organisms do not form adherent biofilms, ${ }^{18}$ while recent tests with marine organisms have shown a decrease in settlement of Ulva linza zoospores and Balanus amphitrite larvae. ${ }^{21}$ These surfaces were made from porous solids infiltrated with a chemicallymatched fluorinated lubricant, but showed results similar to what was observed in this work with silicone oil-infused vascularized PDMS. This supports the hypothesis that the presence of a liquid barrier layer is at least partially contributing to the easy removal of the bacterial and microalgal biofilms in these lubricant-infused samples.

Although the method described here of creating lubricant-infused PDMS with an embedded or encased vascular system may prolong its fouling-release functionality, it does not solve other problems frequently encountered when using PDMS as a fouling-release coating in marine environments, such as low durability and bonding issues. ${ }^{44}$ However, it may also be possible to create vascular networks inside of other types of materials, such as commercially available amphiphilic coatings. ${ }^{44}$ As long as the material being used was capable of being infused with lubricant, such an approach could also increase longevity while not sacrificing durability or adhesion.

\section{CONCLUSIONS}

We have fabricated self-lubricating biofouling-release surfaces designed to mimic natural self-replenishing materials. Samples were created by either encasing a pre-molded vasculature pattern, or by directly embedding channels into uncured PDMS, followed by curing and filling the vascular systems with silicone oil lubricant. Continual replenishment of the silicone oil in the vasculature from an outside source resulted in a surface that could self-lubricate upon oil depletion via simple diffusion of the lubricant from the embedded reservoir. Accelerated tests on lubricant removal via evaporation at $70^{\circ} \mathrm{C}$ showed that the lubricant-infused vascularized systems lost significantly less lubricant over a 24-day period when continuously replenished with lubricant than non-vascularized controls (6.5\% versus $20.8 \%$, respectively).

When challenged with static cultures of $S$. aureus for $48 \mathrm{~h}$, a leading cause of infections in hospital settings, ${ }^{1}$ lubricant-infused PDMS samples showed biofilm coverages of only $0.1 \%$, compared to $74 \%$ and $65 \%$ for glass and non-infused PDMS surfaces, respectively. Colonyforming unit counts of these samples confirmed a decrease of at least three orders of magnitude in the number of bacteria remaining on the infused samples compared to the controls. Tests on a clinically-isolated strain of $E$. coli showed similar results.

Surfaces submerged for 12 days in static cultures of B. braunii, a green microalga known for its potential in the algae biofuels sector, ${ }^{38}$ showed biofilm coverages of $7 \%$ on lubricant- 
infused PDMS substrates as opposed to $98 \%$ and $95 \%$ for glass and non-infused PDMS, respectively. Chlorophyll $a$ values of these samples likewise dropped to $13.4 \mu \mathrm{g} / \mathrm{mL}$ for infused substrates, compared to $269.5 \mu \mathrm{g} / \mathrm{mL}$ for glass and $243.6 \mu \mathrm{g} / \mathrm{mL}$ for non-infused PDMS. Tests on the commercially-used species of $C$. reinhardtii, D. salina, and N. oculata also showed significantly reduced biofilm adherence after removal from the growth medium.

Experiments on lubricant-infused samples kept under accelerated lubricant loss conditions followed by exposure to static cultures of $E$. coli showed a significantly reduced amount of biofilm formation on the vascularized samples versus non-vascularized controls over multiple time points. After $48 \mathrm{~h}$ of exposure to lubricant evaporation conditions, nonvascularized lubricant-infused samples showed $98 \%$ biofilm coverage (equal to non-infused samples), while vascularized controls showed only $5 \%$ coverage.

Tests on the toxicity of the silicone oil used for infusion on cultures of B. braunii revealed no difference in growth rate or live cell numbers upon exposure to excessive amounts of this lubricant over a 14-day period. This eliminated toxicity or cell suffocation as potential mechanisms of action for the fouling-release effects observed.

These results demonstrate the ability of PDMS that is infused with the lubricant postfabrication to resist biofilm attachment from a range of bacterial and microalgal species. They further show that the incorporation of a vascular system can dynamically maintain the foulingrelease properties of these lubricant-infused polymers even in the face of accelerated lubricant loss conditions, mimicking the self-replenishing properties of highly effective and long-lasting living antifouling systems employed by some animals and plants. Although all lubricant infusion in these studies was done using a syringe, these materials could easily be designed to include the incorporation of a lubricant inlet tube for continuous lubricant replenishment from an external reservoir. Furthermore, coatings with incorporated vasculature could be applied dry and then infused with lubricant through the vascular system alone, simplifying the coating process. In summary, the work presented here offers the basis for future dynamic, non-toxic fouling-release coatings with significantly increased longevity arising from the incorporation of a vascular system that provides continuous diffusion of the lubricant to the interfaces.

\section{AUTHOR INFORMATION \\ Corresponding Author}

*Email: chowell@ seas.harvard.edu, jaiz@ seas.harvard.edu

\section{Author Contributions}

The manuscript was written through contributions of all authors. All authors have approved the final version of the manuscript.

\section{Notes}

The authors declare no competing financial interest. 


\section{ACKNOWLEDGEMENTS}

The authors thank Ron Parsons and Joel Butler at Solix Biosystems for providing the N. oculata cultures, and John Skutnik for the D. salina cultures. The authors also thank Shira Lehmann, Bobak Mosadegh and A. Arias Palomo for technical assistance, Isa DuMond for culture assistance, and Ian Burgess for editorial assistance. The leaf vasculature used for the 3D molds was taken by Jon Sullivan (PDPhoto.org) and obtained from the public domain at Wikimedia Commons. The information, data, or work presented herein was funded in part by the by the Office of Naval Research under award no. N00014-11-1-0641 and by the Advanced Research Projects Agency-Energy (ARPA-E) under award no. DE-AR0000326.

\section{REFERENCES}

(1) Guggenbichler, J.; Assadian, O.; Boeswald, M.; Kramer, A. Incidence and Clinical Implication of Nosocomial Infections Associated with Implantable Biomaterials - Catheters, VentilatorAssociated Pneumonia, Urinary Tract Infections Inzidenz Und Kli. GMS Krankenhaushygiene Interdiszip. 2011, 6, 1-19.

(2) Scott, R. D. The Direct Medical Costs of Healthcare-Associated Infections in U.S. Hospitals and the Benefits of Prevention; 2009; pp. 1-16.

(3) Schultz, M. P. Effects of Coating Roughness and Biofouling on Ship Resistance and Powering. Biofouling 2007, 23, 331-341.

(4) Schultz, M. P.; Bendick, J. A.; Holm, E. R.; Hertel, W. M. Economic Impact of Biofouling on a Naval Surface Ship. Biofouling 2011, 27, 87-98.

(5) Singh, R. N.; Sharma, S. Development of Suitable Photobioreactor for Algae Production - A Review. Renewable Sustainable Energy Rev. 2012, 16, 2347-2353.

(6) Van der Bruggen, B.; Cornelis, G.; Vandecasteele, C.; Devreese, I. Fouling of Nanofiltration and Ultrafiltration Membranes Applied for Wastewater Regeneration in the Textile Industry.

Desalination 2005, 175, 111-119.

(7) Chiellini, C.; Iannelli, R.; Modeo, L.; Bianchi, V.; Petroni, G. Biofouling of Reverse Osmosis Membranes Used in River Water Purification for Drinking Purposes: Analysis of Microbial Populations. Biofouling 2012, 28, 969-984.

(8) Tan, S. Y.-E.; Chew, S. C.; Tan, S. Y.-Y.; Givskov, M.; Yang, L. Emerging Frontiers in Detection and Control of Bacterial Biofilms. Curr. Opin. Biotechnol. 2014, 26, 1-6.

(9) Park, K. D.; Kim, Y. S.; Han, D. K.; Kim, Y. H.; Lee, E. H.; Suh, H.; Choi, K. S. Bacterial Adhesion on PEG Modified Polyurethane Surfaces. Biomaterials 1998, 19, 851-859.

(10) Zhou, Z.; Calabrese, D. R.; Taylor, W.; Finlay, J. a; Callow, M. E.; Callow, J. a; Fischer, D.; Kramer, E. J.; Ober, C. K. Amphiphilic Triblock Copolymers with PEGylated Hydrocarbon 
Structures as Environmentally Friendly Marine Antifouling and Fouling-Release Coatings. Biofouling 2014, 30, 589-604.

(11) Mahltig, B.; Fiedler, D.; Boettcher, H. Antimicrobial Sol-Gel Coatings. J. Sol-Gel Sci. Technol. 2004, 32, 219-222.

(12) Kirschner, C. M.; Brennan, A. B. Bio-Inspired Antifouling Strategies. Annu. Rev. Mater. Res. 2012, 42, 211-229.

(13) Magin, C. M.; Cooper, S. P.; Brennan, A. B. Non-Toxic Antifouling Strategies. Mater. Today 2010, 13, 36-44.

(14) Majumdar, P.; Stafslien, S.; Daniels, J.; Webster, D. C. High Throughput Combinatorial Characterization of Thermosetting Siloxane-urethane Coatings Having Spontaneously Formed Microtopographical Surfaces. J. Coat. Technol. Res. 2007, 4, 131-138.

(15) Genzer, J.; Efimenko, K. Recent Developments in Superhydrophobic Surfaces and Their Relevance to Marine Fouling: A Review. Biofouling 2006, 22, 339-360.

(16) Baum, C.; Meyer, W.; Stelzer, R.; Fleischer, L.; Siebers, D. Average Nanorough Skin Surface of the Pilot Whale (Globicephala Melas , Delphinidae): Considerations on the Self-Cleaning Abilities Based on Nanoroughness. Mar. Biol. 2002, 140, 653-657.

(17) Wong, T.-S.; Kang, S. H.; Tang, S. K. Y.; Smythe, E. J.; Hatton, B. D.; Grinthal, A.; Aizenberg, J. Bioinspired Self-Repairing Slippery Surfaces with Pressure-Stable Omniphobicity. Nature 2011, 477, 443-447.

(18) Epstein, A. K.; Wong, T.-S.; Belisle, R. a; Boggs, E. M.; Aizenberg, J. Liquid-Infused Structured Surfaces with Exceptional Anti-Biofouling Performance. Proc. Natl. Acad. Sci. U. S. A. 2012, 1-6.

(19) Yao, X.; Dunn, S. S.; Kim, P.; Duffy, M.; Alvarenga, J.; Aizenberg, J. Fluorogel Elastomers with Tunable Transparency, Elasticity, Shape-Memory, and Antifouling Properties. Angew. Chem. Int. Ed. Engl. 2014, 53, 4418-4422.

(20) Li, J.; Kleintschek, T.; Rieder, A.; Cheng, Y.; Baumbach, T.; Obst, U.; Schwartz, T.; Levkin, P. A. Hydrophobic Liquid-Infused Porous Polymer Surfaces for Antibacterial Applications. ACS Appl. Mater. Interfaces 2013, 5, 6704-6711.

(21) Xiao, L.; Li, J.; Mieszkin, S.; Di Fino, A.; Clare, A. S.; Callow, M. E.; Callow, J. a; Grunze, M.; Rosenhahn, A.; Levkin, P. a. Slippery Liquid-Infused Porous Surfaces Showing Marine Antibiofouling Properties. ACS Appl. Mater. Interfaces 2013, 5, 10074-10080.

(22) Banerjee, I.; Pangule, R. C.; Kane, R. S. Antifouling Coatings: Recent Developments in the Design of Surfaces That Prevent Fouling by Proteins, Bacteria, and Marine Organisms. Adv. Mater. 2011, 23, 690-718.

(23) Friedlander, R. S.; Vlamakis, H.; Kim, P.; Khan, M.; Kolter, R.; Aizenberg, J. Bacterial Flagella Explore Microscale Hummocks and Hollows to Increase Adhesion. Proc. Natl. Acad. Sci. U. S. A. 2013, 110, 5624-5629. 
(24) Brady, R. F.; Singer, I. L. Mechanical Factors Favoring Release from Fouling Release Coatings. Biofouling 2000, 15, 73-81.

(25) Liu, K.; Jiang, L. Bio-Inspired Self-Cleaning Surfaces. Annu. Rev. Mater. Res. 2012, 42, 231-263.

(26) Baier, R. Substrata Influences on Adhesion of Microorganisms and Their Resultant New Surface Properties. In Adsorption of Microorganisms to Surfaces; Bitton, G.; Marshal, K., Eds.; John Wiley and Sons: New York, 1980; pp. 59-104.

(27) Nendza, M. Hazard Assessment of Silicone Oils (polydimethylsiloxanes, PDMS) Used in Antifouling-/foul-Release-Products in the Marine Environment. Mar. Pollut. Bull. 2007, 54, 11901196.

(28) Kavanagh, C. J.; Swain, G. W.; Kovach, B. S.; Stein, J.; Darkangelo-Wood, C.; Truby, K.; Holm, E.; Montemarano, J.; Meyer, A.; Wiebe, D. The Effects of Silicone Fluid Additives and Silicone Elastomer Matrices on Barnacle Adhesion Strength. Biofouling 2003, 19, 381-390.

(29) Truby, K.; Wood, C.; Stein, J.; Cella, J.; Carpenter, J.; Kavanagh, C.; Swain, G.; Wiebe, D.; Lapota, D.; Meyer, a; Holm, E.; Wendt, D.; Smith, C.; Montemarano, J. Evaluation of the Performance Enhancement of Silicone Biofouling-Release Coatings by Oil Incorporation. Biofouling 2000, 15, 141-150.

(30) Scardino, A. J.; de Nys, R. Mini Review: Biomimetic Models and Bioinspired Surfaces for Fouling Control. Biofouling 2011, 27, 73-86.

(31) Ganguli, R.; Mehrotra, V.; Dunn, B. Bioinspired Living Skins for Fouling Mitigation. Smart Mater. Struct. 2009, 18, 104027.

(32) Wu, W.; DeConinck, A.; Lewis, J. A. Omnidirectional Printing of 3D Microvascular Networks. Adv. Mater. 2011, 23, H178-83.

(33) Rivas, M. O.; Vargas, P.; Riquelme, C. E. Interactions of Botryococcus Braunii Cultures with Bacterial Biofilms. Microb. Ecol. 2010, 60, 628-635.

(34) Vollenweider, R. IBP Handbook No. 12: A Manual on Methods for Measuring Primary Production in Aquatic Environments; F.A. Davis Co.: Philadelphia, PA, 1969; pp. 28-40.

(35) Sato, M.; Murata, Y.; Mizusawa, M.; Iwahashi, H.; Oka, S. A Simple and Rapid Dual Fluorescence Viability Assay. Mircobiol. Cult. Coll. 2004, 20, 53-59.

(36) Zar, J. H. Biostatistical Analysis; 4th Editio.; Prentice Hall: Upper Saddle River, NJ, 1993; p. 279.

(37) Casadevall, E.; Dif, D.; Largeau, C.; Gudin, C.; Chaumont, D.; Desanti, O. Studies on Batch and Continuous Cultures of Botryococcus Braunii: Hydrocarbon Production in Relation to Physiological State, Cell Ultrastructure, and Phosphate Nutrition. Biotechnol. Bioeng. 1985, 27, 286-295.

(38) Duong, V. T.; Li, Y.; Nowak, E.; Schenk, P. M. Microalgae Isolation and Selection for Prospective Biodiesel Production. Energies 2012, 5, 1835-1849. 
(39) Zou, N.; Richmond, A. Effect of Light-Path Length in Outdoor Flat Plate Reactors on Output Rate of Cell Mass and of EPA in Nannochloropsis Sp. J. Biotechnol. 1999, 70, 351-356.

(40) Solovchenko, A.; Khozin-Goldberg, I.; Recht, L.; Boussiba, S. Stress-Induced Changes in Optical Properties, Pigment and Fatty Acid Content of Nannochloropsis Sp.: Implications for NonDestructive Assay of Total Fatty Acids. Mar. Biotechnol. (NY). 2011, 13, 527-535.

(41) Stevens, C.; Powell, D. E.; Petteri, M.; Karman, C. Fate and Effects of Polydimethylsiloxane (PDMS) in Marine Environments. Mar. Pollut. Bull. 2001, 42, 536-543.

(42) Graiver, D.; Farminer, K. W.; Narayan, R. A Review of the Fate and Effects of Silicones in the Environment. J. Polym. Environ. 2003, 11, 129-136.

(43) Helena, L.; Rodrigues, R.; Arenzon, A.; Raya-rodriguez, M. T.; Fontoura, N. F. Algal Density Assessed by Spectrophotometry: A Calibration Curve for the Unicellular Algae Pseudokirchneriella Subcapitata. J. Environ. Chem. Ecotoxicol. 2011, 3, 225-228.

(44) Callow, J. A.; Callow, M. E. Trends in the Development of Environmentally Friendly FoulingResistant Marine Coatings. Nat. Commun. 2011, 2, 244.
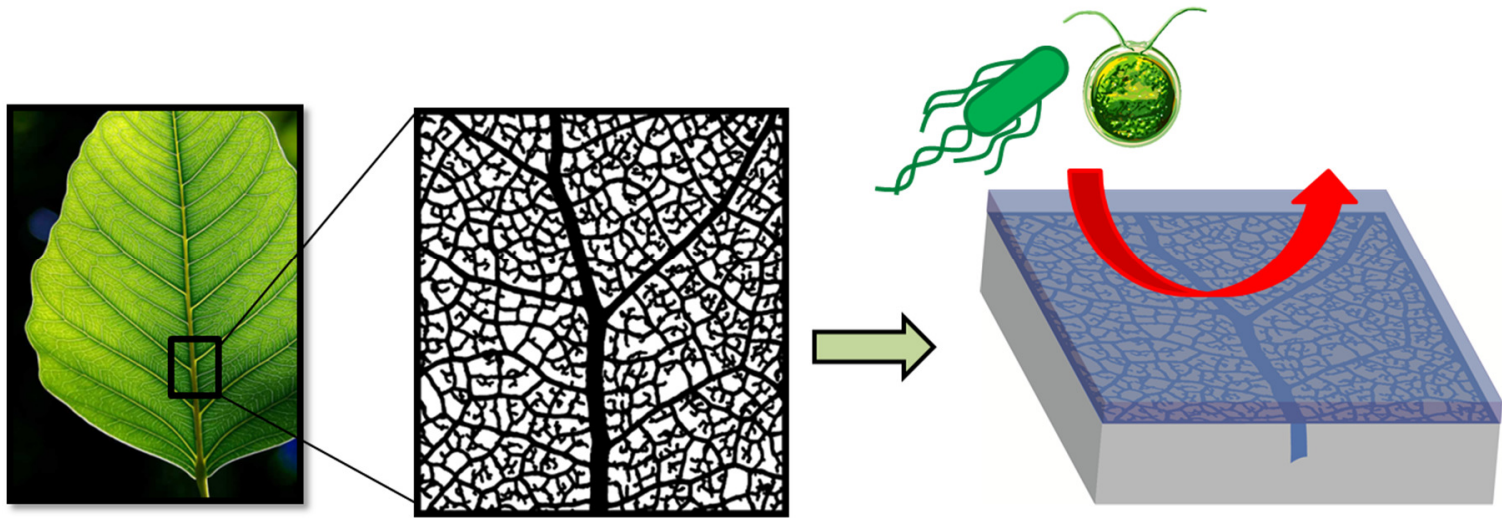

Table of Contents Graphic. 